\title{
Acidic Skin Care Promotes Cutaneous Microbiome Recovery And Skin Physiology in An Acute Stratum Corneum Stress Model
}

Joachim W. Fluhr ( $\square$ joachim.fluhr@charite.de)

Charité Universitätsmedizin Berlin Campus Charite Mitte: Charite Universitatsmedizin Berlin https://orcid.org/0000-0003-3610-0698

\section{Peter Menzel}

Labor Berlin

\section{Rolf Schwarzer}

Labor Berlin

Benjamin Kaestle

Sebapharma

Michaela Arens-Corell

Sebapharma

Lina Praefke

Sebaphamra

Nikolai Tsankov

Acibadem University: Acibadem Universitesi

Dessislava G. Nikolaeva

Acibadem, Tokudy University Hospital, Sofia

\section{Laurent Miséry}

Univerité de Brest

\section{Razvigor Darlenski}

Univ. Stara Zagora

\section{Research}

Keywords: microbiome, skin physiology, acidic skin care

Posted Date: November 1st, 2021

DOI: https://doi.org/10.21203/rs.3.rs-1016070/v1

License: (a) (i) This work is licensed under a Creative Commons Attribution 4.0 International License.

Read Full License 


\section{Abstract}

\section{Background:}

The skin microbiome and skin physiology are important indicators of the epidermal homeostasis status. Stress models are used to reveal pathological conditions and modulating effects. The recovery phase served to investigate the cutaneous microbiome in relation to skin physiology after mild tape stripping without treatment compared to two cosmetic leave-on lotions ( $\mathrm{pH} 5.5$ vs. $\mathrm{pH}$ 9.3).

\section{Material and Methods}

The prospective, randomized, controlled study was performed in 25 healthy volunteers: The microbiome was assessed via swabs and subsequent 16S-rRNA-gene amplicon sequencing. Skin physiology was analyzed in terms of barrier function, stratum corneum hydration, surface-pH, skin color non-invasively. All parameters were assessed before and immediately after $2 \mathrm{hrs}$., 2 days and 7 days after tape stripping. Lotion $\mathrm{A}(\mathrm{pH}$ 5.5) and the identical lotion $\mathrm{B}(\mathrm{pH}$ 9.3) were applied 2-times per day for 7 days on the volar forearm.

\section{Results}

Tape stripping reduced the alpha diversity with a recovery over 7 days without treatment. Both lotions significantly accelerated the recovery of the alphadiversity already after 2 days with a slightly higher rate for lotion A ( $\mathrm{pH}$ 5.5). After tape stripping, the relative abundance of Proteobacteria was increased, whereas Actinobacteria were reduced. Further, mean relative abundances of typical skin-associated genera were reduced after tape stripping. Taxa compositions returned to normal levels after 7 days in all treatment groups, and an accelerated normalization could be observerd with treatment by both lotions already after tow days. A significant difference in skin-pH was observed at day2 and day7 with an increased $\mathrm{pH}$ in lotion $\mathrm{B}$ treatment. Both lotions induced an increase in stratum corneum hydration. Barrier function was only changed by tape stripping.

\section{Discussion}

The study proved the suitability of an experimental stress model in the assessment of skin surface microbiome in relation to skin physiology. Stratum corneum hydration increased significantly with both lotions already at day 2. Microbiome parameters (alpha-diversity, mean relative taxa, abundance of selected genera) normalized over 2-7 days. Three potential mechanisms could be responsible for the accelerated normalization: a) optimized hydration during the recovery phase b) the composition of the lotion, $\mathrm{c}$ ) acidic $\mathrm{pH}$ of the lotion.

\section{Introduction}

Cutaneous microbiome and skin physiology are important indicators of the epidermal homeostasis status. The microbial bio-diversity in the upper epidermis is, influenced by the interaction with exogenous 
factors, eg, the environment encompassing exposure to a specific professional or leisure activity or skin care habit [1, 2]. Furthermore, exogenous stressors like mechanical abrasion, chemical irritants and UVirradiation have an impact on the homeostasis of functional parameters and the microbiome [2,3].

The complex interaction and variability of exogenous factors influencing the homeostasis of an organism is encompassed by the term "exposome" [4]. Life style factors like food with a high content of anti-oxidative ingredients, living with a partner or a dog, as well as smoking, hygiene procedures, prescription drugs (e.g. antibiotics and immuno-suppressants) have been shown to influence both skin physiology $[5,6]$, and the microbiome $[7,8]$. Thus, studies that have an integrated look both at skin physiology and the microbiome might reveal the relation between the homeostasis of functional skin parameters and changes in the microbiome.

The importance of the epidermal acid mantle has been known for several decades [9-11]. The current understanding regarding the origin of the acid buffer system of the $\mathrm{SC}$ is mainly based on phospholipid/free fatty acid, histidase and $\mathrm{Na}+-\mathrm{H}+$ exchanger-1 (NHE1) pathways [12]. The acidic skin $\mathrm{pH}$ resides in the upper stratum corneum and shows a gradient towards neutralization in deeper part of the epidermis in relation to active, energy requiring mechanisms involved in keratinocyte differentiation processes [13]. The consequences of an alteration in epidermal functions associated with an elevated skin $\mathrm{pH}$ can be observed in inflammatory skin diseases, under occluded and hyperhydrated SC and skin infections such as candidiasis $[14,15]$.

Supporting the acid mantle is well established in skin care and an acidic $\mathrm{pH}$ is an important parameter in leave on and rinse off skin care products [16]. Furthermore, acidic pH skin care has been shown to exert positive effects on skin physiology and specific microbial colonization patterns, especially in the context of atopic dermatitis $[15,17][18]$

The skin microbiome has been subject to intense research over the last decade: First the skin microbiome has been characterized, with specific patterns in highly hydrated areas as well as in sebum rich areas [19]. The relation of sebum and hydration levels on microbiome diversity was confirmed specifically in the facial region [20]. Finally, the use of an emollient in infants at risk for developing AD decreased the surface $\mathrm{pH}$ and subsequently increased the proportion of $S$. salivarius [21].

Stress models are used in assessing barrier-related parameters to reveal pathological conditions and modulating effects during the recovery phase $[22,23]$. This so-called 'epidermal treadmill stress model' helped to reveal underlying pathophysiological mechanisms of many skin diseases with altered epidermal functions [24]. The tape stripping (TS) stress model was adapted to microbiome research. Microbiome dynamics of the epidermis in human volunteers upon an acute barrier disruption by sequential TS has been described [25]. A regeneration of the diversity after acute removal from superficial parts of the SC induces active repair processes. Bacteria DNA was evidenced using independent detection techniques down to the dermis and the dermal adipose tissue of normal skin of humans [26]. 
In the present study, we were interested in short-term and mid-term recovery of skin physiology in relation to the microbiome after mild TS in treatments with two cosmetic leave-on lotions (pH 5.5 vs. pH 9.3) compared to untreated areas.

We hypothesized that i) that barrier disruption with a mild acute stress model would induce significant changes in skin physiology, ii) the stress model would lead to an immediate decrease in the diversity of the microbiome, iii) the recovery of both skin physiology and microbiome alteration could be accelerated with leave-on skin care, iv) an acidic $\mathrm{pH}$ of the leave-on lotion would be favourable for the recovery of skin physiology and the microbiome parameters.

\section{Material And Methods}

\section{Study population}

The study was approved by the institutional ethics board of the Ethical Committee of the Euroderma Clinic in Sofia from 9.07.2020 with protocol number 2/9.7.2020. All volunteers gave their written informed consent prior to the start of the study. 25 healthy volunteers (mean age, gender?) were included and completed the study. The statistical analysis was performed with Prism 6. The male-female ratio was 10:15; mean age 30.7 years (SEM 2.1), BMI 21.7 (SEM 0.8), smoker: non-smoker ratio 12:13.

\section{Design of the study}

Initial values on stratum corneum hydration, surface $\mathrm{pH}$, skin color and barrier function as well as microbiome analysis were performed at baseline. Tape stripping was carried out with 5 sequential strippings with Corneofix ${ }^{\circledR}$ applied with standardized pressure of $225 \mathrm{gr} / \mathrm{cm}^{2}$ for 5 seconds each $[27,28]$. Three different areas on the volar forearms were tape-stripped, one remained the untreated control area. Definition of the sites was achieved by using a template adjusted to the inner fold of both elbows for measurements and microbiome swabs during the study. Skin color, barrier function and microbiome swabs were obtained immediately after tape stripping as well as $2 \mathrm{~h}$ later. Further measurements of the skin physiology parameters and microbiome swab sampling were performed after 2 days (48 hrs.) and 7 days (168 hrs.).

\section{Skin Physiology Parameters}

Epidermal barrier function was evaluated with the open-chamber system Tewameter TM300. The values are given in $\mathrm{g} / \mathrm{m}^{2} \mathrm{hr}$. Stratum corneum hydration was measured with Corneometer CM 825. The values are given in arbitrary units (AU). Surface $\mathrm{pH}$ was quantified with the $\mathrm{pH}-$ Meter including a flat-glass electrode from Mettler-Toledo. The values are given in pH-units. Skin color was measured with the Mexameter MX 18 with the erythema index and the melanin index; both in arbitrary units. All instruments are manufactured by Courage\&Khazaka electronics (Cologne, Germany). All probes were attached to a central unit (multiprobe adaptor MPA-9) and subsequently to a PC. The published guidelines were 
respected $[29,30]$. The acclimatization time at constant temperature $\left(21^{\circ} \mathrm{C}\right)$ and air humidity $(55 \%)$ prior to the measurements of skin parameters was between 20 and 30 minutes.

\section{Test formulations}

Lotion A with a pH of 5.5 (range 5.2 - 5.8) (named lotion A) and a product of the same composition with a pH of 9.3 (range 9.1 - 9.5; adjusted and buffered by the addition of Bicarbonate and Sodium Hydrogencarbonate) (named lotion B) were compared. The INCI list of lotion ingredients was as following: Aqua, Glycerin, Cetearyl Alcohol, Sorbitol, Hexyldecanol, Hexyldecyl Laurate, Chamomilla recutita flower extract, Allantoin, Sodium Cetearyl Sulfate, Citric Acid, Sodium Hydroxide, Sodium Acetate, Sodium Ascorbate, Xanthan Gum, Dimethicone, Parfum, Alcohol, Phenoxyethanol, Sodium Benzoate, Benzyl Alcohol.

Lotion $A$ and $B$ treatment areas as well as TS-untreated and non-TS untreated areas were assigned with a randomization list. A fingertip unit of lotion $A$ and $B$, respectively, was applied to the assigned test areas twice daily and rubbed in until they were completely absorbed for 6 consecutive days.

Washing of the forearms, showering or bathing with water was allowed only $24 \mathrm{~h}$ before and not at all 3 hours prior to the baseline examination. No skin care products were to be applied on the forearms $24 \mathrm{~h}$ prior to the baseline examination. $12 \mathrm{~h}$ before the analysis on day 2 and day 7 no washing of the forearms, showering or bathing with water or use of any cosmetic products including the test products, was allowed.

\section{Microbiome Sequencing}

DNA was extracted and purified into 50 $\mu$ l elution buffer using the DNeasy PowerSoil Pro-Kit (Qiagen). 2.6 pg of DNA from Salinibacter ruber (DSM 13855) was added as an internal spike-in control. Amplification of the V3-V4 region of the 16S rRNA gene was done with the UCP multiplex PCR mastermix (Qiagen) from $5 \mu$ INA using the primers CCTACGGGNGGCWGCAG (forward) and GACTACHVGGGTATCTAATCC (reverse) following the Illumina 16S library preparation protocol

(https://support.illumina.com/documents/documentation/chemistry_documentation/16s/16smetagenomic-library-prep-guide-15044223-b.pdf). Sequencing was done on an Illumina MiSeq (v2 reagents) with $2 \times 250 \mathrm{bp}$ paired-end reads.

\section{Sequence Analysis}

After sequencing, the UPARSE 16S-protocol was used to merge the paired reads by their 3 '-ends, filter reads, and cluster unique reads into OTUs using a $97 \%$ identity threshold, and quantify OTU abundances by mapping reads to the OTU sequences[31]. OTUs with a relative abundance of less than $0.01 \%$ were discarded. Each OTU sequence was searched in the NCBI Targeted Loci 16S database using NCBI BLAST[32] with an identity cut-off of $97 \%$ and E-value cut-off of 0.01 , and assigned to the annotated species. If no match was found, the sequence was also searched in the NCBI nt database using the same 
cut-offs but also excluding unclassified species or environmental sequences. If no match was found in either database, the OTU was labelled as unclassified. OTUs with multiple best matches were assigned to the least common ancestor of all alignments with the same BLAST bit score. OTUs assigned to the Salinibacter ruber spike-in were discarded.

Alpha diversity is measured as the number of OTUs in each sample. Relative abundances for species were calculated from classified OTUs and summarized on taxonomic ranks from species to phylum. Visualisation of microbiome data was done using ggplot2[33].

\section{Statistical analysis:}

Biophysical analysis: One way ANOVA analysis within time groups was calculated followed by Sidak's multiple comparisons test. The significance level was set at $p<0.05$.

Microbiome: Comparison of diversity between treatment groups was performed with the Durbin-Conover test and Holm multiple testing correction using ggstatsplot[34].

\section{Results}

\section{Validity of the epidermal barrier stress model}

TEWL and erythema index were assessed immediately after and 120 min post TS procedure to evaluate the validity of epidermal barrier disruption. As shown in figure 1B. A significant increase immediately after $(p<0.001 * \star * a)$ and $120 \mathrm{~min}(p<0.01 * * b)$ after TS procedure was revealed, confirming the validity of this in vivo stress model of skin barrier disruption. No variations were noted for melanin and erythema index (Figure S4) indicating that the stress model was of mild intensity since no clinically visible or measurable erythema was induced. The mean room temperature at the three measurement time points was between $19.7^{\circ} \mathrm{C}$ and $20.2^{\circ} \mathrm{C}$; the mean relative humidity varied between 44.1 and $46.7 \%$; both parameters showing values in accordance with published guidelines $[29,30]$.

\section{Skin physiology dynamics following product application}

A significant increase in stratum corneum hydration $(\mathrm{SCH})$ was observed on day 2 and day 7 in comparison to untreated skin sites for both lotions. The change in $\mathrm{SCH}$ upon product application is shown in figure 2. Lotion A showed slightly higher values compared to lotion B without reaching statistical significance.

A clear difference in skin surface $\mathrm{pH}$ was observed at both day 2 and day 7 (figure 3). Lotion B application resulted in a significant increase in $\mathrm{pH}$ both at day 2 and day 7 in comparison to Lotion $\mathrm{A}$ and untreated, tape stripped skin area. Skin surface pH did not differ between the area treated with Lotion $\mathrm{A}$ and untreated tape stripped site. 
No significant difference between sites in TEWL (figure 1A), Erythema- and Melanin-index (Figure S4) could be revealed at day 2 and 7 .

\section{Microbiome analysis}

Data from 24 out of the 25 individuals were analysed, one individual was excluded because of missing samples.

The number of sequencing reads per sample ranged from 1,117 to 168,807 (mean 20,7764, median 11356) and was on average higher in the treated samples and lowest in untreated samples after tape stripping (Supplement Figure S1). A comparison between the fraction of numbers of operational taxonomy units (OTUs) that are classified to a taxon and the fraction of reads belonging to classified OTUs shows that on average $83 \%$ of OTUs are classified, but they comprise on average $95 \%$ of the reads, i.e., most unclassified OTUs are of low abundance (Supplement Figure S2). The rarefaction analysis (Supplement Figure S3) shows the number of OTUs for the 11 samples per individual after subsampling to half read depth, one fourth read depth and further dilutions down to 1/1024. Rarefaction curves reached a plateau in all samples, with at least $95 \%$ of OTUs present at half read depth.

The alpha-diversity was unchanged during the 7 days of the study in the non-stripped and non-treated control areas (figure 4a), with mean values from 183 to 204 . Tape stripping significantly reduced the alpha-diversity to 125. The values increased in the untreated areas to 177 after 2 days and 228 after 7 days (figure 4b). Both lotions significantly accelerated the recovery of the alpha-diversity already at 2 days compared with the untreated areas to a mean of 281 for lotion A and 272 for lotion B (figure 4c). After 7 days no significant differences between the groups were found, but we observe a decrease of alpha diversity compared with the measurements from 2 days after tape stripping in both lotions to levels comparable with the untreated areas (figure $\mathbf{4 d}$ ).

The distribution of phyla (figure 5a) was unchanged in the control area over the 7 days of the study. After tape stripping, the relative abundance of Proteobacteria was increased, whereas Actinobacteria abundances decreased. This shift from Actinobaceria towards Proteobacteria recovered partially after 2 days and normalized almost completely at day 7 . Treatment with lotion $A$ and lotion $B$ accelerated the normalization: Already after 2 days the distribution was comparable to the baseline non-stripped area and remained similar on day 7. Examination of the most abundant orders revealed an increase of Burkholderiales after tape stripping as well as a reduction in particular of the lower abundant orders like Corynebacteriales, Micrococcales, Propionibacteriales, and Lactobacillales, with the exception of Xanthomonadales, where the relative abundance increased (figure 5B). Abundances of Bacillales and Pseudomonadales remained largely unchanged.

The mean relative abundances of the 20 most abundant genera in each group are shown in figure 6 . The first row shows control areas, the second row the TS / untreated area, and the third and fourth row show lotions $A$ and $B$ respectively. The control group showed a stable genus composition over the whole period, with Staphylococcus as the dominant genus. TS induced a relative increase of brevibacillus and a 
reduction of staphylococci and corynebacteria. After 7 days the genus composition was again similar to the control areas. Treatment with both lotions resulted in normalization of the genus distribution already at day 2 .

Noteworthy is a major reduction of the four typical skin-associated genera Corynebacterium, Cutibacterium, Staphylococcus and Streptococcus after tape stripping (figure 7a). Both lotions accelerated their recovery to normal and even elevated levels compared with control and untreated groups. When plotting the combined relative abundance of these four genera against the alpha diversity in each sample (figure 7b), we observe no change over time in control areas, but a shift towards low abundance and low alpha diversity in the TS areas. The groups of both lotions show a similar distribution as in the control group and no relevant shift of both variables between the two time points, indicating that lotion treated skin areas returned to almost normal skin flora composition already after two days.

Figure 8 shows the mean relative abundance of the OTUs that are assigned to different Staphylococcus species (S. aureus / S. simiae, S. capitis / S. caprae, S. capitis / S. caprae / S. epidermidis, as well as summarized other Staphylococcus spp.) and the number of samples in which they were observed. TS induced a similar reduction in all Staphylococcus OTUs. This reduction returned to almost normal levels after 2 days but only after 7 days showed comparable abundances to untreated areas.

\section{Discussion}

Exogenous environmental influences are known to alter skin physiology and microbiome parameters in humans [1-3]. We hypothesized that, an acute epidermal stress model would be suitable to detect the positive effect of an acidic skin care on recovery dynamics both of skin physiology and epidermal microbiome diversity and other microbiome parameters. The TS stress model is an established model to reveal subtle differences in pathological states as well as for specific treatment modalities $[35,36]$. TS was also described to serve as a model in studying skin microbiome [25,37]. We selected a mild TS model in healthy volunteers which showed to be correctly executed with mild barrier disruption but no visible or measurable irritation.

The recovery of skin physiology was accelerated by skin care, however not in a pH-dependent manner. Both lotions (only differing in the $\mathrm{pH}$-related buffer substances) revealed a positive effect on barrierrelated parameters. Only for skin surface $\mathrm{pH}$ a significant difference was observed at day 2 and day 7 with an increased $\mathrm{pH}$ induced by basic/alkaline $\mathrm{pH}$ of lotion $\mathrm{B}$. No significant differences between sites were detectable in barrier function (TEWL) and irritation (erythema index) at day 2 and day 7 .

TS significantly reduced the alpha-diversity, followed by a recovery over 7 days without treatment. Thus our assumption of TS being a relevant model for studying the dynamics of microbial recovery after superficial removal of stratum corneum was confirmed. The re-population of the stripped skin section is coming both from lateral ingrowth and from preserved, deeper parts of the skin and hair follicles [25] . 
Both lotions accelerated the recovery of the microbiome, showing a significantly higher alpha diversity already after 2 days, with a slightly higher induction rate for lotion $\mathrm{A}$ (lower $\mathrm{pH}$ ). After 7 days, the alpha diversity slightly decreased again and it was similar to the control areas and untreated stripped areas. This suggests that the repopulation of stripped area started with an initial overcompensation caused by a replacement flora that rapidly fills the empty niche. After a few days, the normal flora replaces this transitional microbiome.

After TS, the abundance of Burkholderiales increased, whereas abundances of several other genera decreased. In particular, the abundance of the common skin-associated genera Staphylococcus, Corynebacterium, Streptococcus and Cutibacterium was reduced after TS. Similar to the alpha-diversity, the shift in the microbiome composition returned to a normal state over the course of 7 days in the untreated group. Topical treatment with lotions accelerated the normalization of the taxa distribution, and already after 2 days the distribution was comparable to the control none-stripped areas and remained similar on day 7.

The inter-individual, sometimes large differences in species distribution between the volunteers (baseline levels) represents a limiting factor when interpreting microbiome data. The high variation makes it challenging to identify significant and relevant effects induced by $\mathrm{pH}$ changes since they might be masked by the inter-individual variation in the present cohort size.

The present study confirmed the value of skin physiology assessment in an experimental stress model, namely barrier disruption by TS. The validity of this model has been proven as shown in figure 1 . This model was used to evaluate the effect of the $\mathrm{pH}$ of a marketed cosmetic product on the epidermal barrier recovery and skin physiology parameters. No significant change in TEWL at day 2 and day 7 was registered neither between both lotion-treated sites nor between untreated, TS area and untreated unstripped site. A probable limitation is the spontaneous barrier recovery within 2 days after TS, reflected by the non-significant difference between untreated TS, and untreated unstripped area already at day 2 . Our stress-model of a single barrier damage only induced a mild barrier damage with a relatively fast barrier recovery [24]. A more pronounced increase in TEWL or repetitive damage would have caused more severe disturbances, with a longer regeneration phase. The chosen mild barrier disturbance corresponds to the everyday situations.

$\mathrm{SCH}$ increased significantly under both treated areas (lotions $\mathrm{A}$ and $\mathrm{B}$ ) already at day 2 and was still higher at day 7 in comparison to untreated sites. This cannot be attributed to remnants of the cosmetic product itself as volunteers were asked not to apply study products 12 hours before measurements at day 2 and 7. Micro-morphological changes were detectable in a multi-ethnic study-population treated for 14 days with an acidic lotion by optical coherence tomography and reflectance confocal microscopy [38].

Published studies showed that a change in hygiene routines has only limited effects on the microbiome. Changes in the microbiome were only detected after usage of an antiperspirant and foot powder, yet no changes were observed after usage of lotions [7, 39]. 
Our findings proved the applicability of an experimental stress model (TS) in the assessment of skin surface microbiome homeostasis in combination with skin physiology. $\mathrm{SCH}$ increased significantly after treatment with both lotions already at day 2 and was still higher at day 7 in comparison to untreated sites. The normalization of the stressed microbiome parameters (including alpha-diversity, mean relative taxa distribution on the phylum-level, abundance of selected genera) was already detectable after $2 \mathrm{hrs}$. and the parameters returned to normal after 7 days of treatment.

Together, the initial hypothesis could be answered as following: i) that barrier disruption with a mild acute stress model induced significant changes in skin physiology, measured as TEWL without leading to a relevant inflammation ii) the stress model lead to an immediate decrease in the diversity of the microbiome, iii) the recovery of both skin physiology and microbiome alteration were accelerated by leave-on skin care, iv) a significant superiority of the acidic $\mathrm{pH}$ lotion compared to the basic/alkaline $\mathrm{pH}$ lotion could not be shown during the recovery of skin physiology and the microbiome parameters.

Three potential mechanisms could be responsible for the accelerated normalization: a) the composition of the lotion, $\mathrm{b}$ ) acidic $\mathrm{pH}$ of the lotion, $\mathrm{c}$ ) optimized hydration during the recovery phase. Further studies should be performed with different stress models as well as a prolonged application time of the acidic skin care.

\section{Declarations}

\section{Data availability}

The sequencing data for all 264 microbiome samples are available at NCBI Genbank under Bioproject accession number PRJNA713953.

- Ethics approval and consent to participate: The study was appoved by the institutional ethics board of the Ethical Committee of the Euroderma Clinic in Sofia from 9.07.2020 with protocol number 2/9.7.2020. All volunteers signed a written informed consent.

- Consent for publication: All authors have read the manuscript, agreed on its submission and consented the publication

- Availability of data and material: All data generated or analysed during this study are included in this published article [and its supplementary information files]. The sequencing data for all 264 microbiome samples are available at NCBI Genbank under Bioproject accession number PRJNA713953.

- Competing interests: JWF and RD have received consulting fees from Sebapharma. BK, MAC and LI are employees of Sebapharma

- Funding: This study was sponsored by Sebapharma, Boppard, Germany

- Authors' contributions

Conception of the study: JWF, RD, NT, LM, MAC, LP, BK 
Performing the study: DGN, JWF; RD

Microbiome analysis: PM, RS

Data analysis: JWF, PM, RS, DGN, RD

Interpretation of the data: all authors

Writing of the manuscript: all authors

- Acknowledgements: We would like the volunteers for their dedicated participation in the study.

\section{References}

1. Khmaladze I, Leonardi M, Fabre S, Messaraa C, Mavon A: The Skin Interactome: A Holistic "GenomeMicrobiome-Exposome" Approach to Understand and Modulate Skin Health and Aging. Clin Cosmet Investig Dermatol 2020, 13:1021-1040.

2. Boxberger M, Cenizo V, Cassir N, La Scola B: Challenges in exploring and manipulating the human skin microbiome. Microbiome 2021, 9:125.

3. Sfriso R, Claypool J: Microbial Reference Frames Reveal Distinct Shifts in the Skin Microbiota after Cleansing. Microorganisms 2020, 8.

4. Wild CP: Complementing the genome with an "exposome": the outstanding challenge of environmental exposure measurement in molecular epidemiology. Cancer Epidemiol Biomarkers Prev 2005, 14:1847-1850.

5. Percoco G, Patatian A, Eudier F, Grisel M, Bader T, Lati E, Savary G, Picard C, Benech P: Impact of cigarette smoke on physical-chemical and molecular proprieties of human skin in an ex vivo model. Exp Dermatol 2020.

6. Barresi R, Chen E, Liao IC, Liu X, Baalbaki N, Lynch S, Brieva P, Wang M, Zheng Q, Bouez C: ARTICLE: Alteration to the Skin Barrier Integrity Following Broad-Spectrum UV Exposure in an Ex Vivo Tissue Model. J Drugs Dermatol 2021, 20:23s-28s.

7. Pinto D, Ciardiello T, Franzoni M, Pasini F, Giuliani G, Rinaldi F: Effect of commonly used cosmetic preservatives on skin resident microflora dynamics. Sci Rep 2021, 11:8695.

8. Nichols RG, Peters JM, Patterson AD: Interplay Between the Host, the Human Microbiome, and Drug Metabolism. Hum Genomics 2019, 13:27.

9. Fluhr JW, Kao J, Jain M, Ahn SK, Feingold KR, Elias PM: Generation of free fatty acids from phospholipids regulates stratum corneum acidification and integrity. J Invest Dermatol 2001, 117:44-51.

10. Fluhr JW, Darlenski R: Skin Surface pH in Newborns: Origin and Consequences. Curr Probl Dermatol 2018, 54:26-32. 
11. Kleesz P, Darlenski R, Fluhr JW: Full-body skin mapping for six biophysical parameters: baseline values at 16 anatomical sites in 125 human subjects. Skin Pharmacol Physiol 2012, 25:25-33.

12. Fluhr JW, Elias PM, Man MQ, Hupe M, Selden C, Sundberg JP, Tschachler E, Eckhart L, Mauro TM, Feingold KR: Is the filaggrin-histidine-urocanic acid pathway essential for stratum corneum acidification? J Invest Dermatol 2010, 130:2141-2144.

13. Damen M, Wirtz L, Soroka E, Khatif H, Kukat C, Simons BD, Bazzi H: High proliferation and delamination during skin epidermal stratification. Nat Commun 2021, 12:3227.

14. Kuo SH, Shen CJ, Shen CF, Cheng CM: Role of pH Value in Clinically Relevant Diagnosis. Diagnostics (Basel) 2020, 10.

15. Schmid-Wendtner $\mathrm{MH}$, Korting HC: The $\mathrm{pH}$ of the skin surface and its impact on the barrier function. Skin Pharmacol Physiol 2006, 19:296-302.

16. Gustin J, Bohman L, Ogle J, Chaudhary T, Li L, Fadayel G, Mitchell MC, Narendran V, Visscher MO, Carr AN: Use of an emollient-containing diaper and pH-buffered wipe regimen restores skin pH and reduces residual enzymatic activity. Pediatr Dermatol 2020, 37:626-631.

17. Hulpusch C, Tremmel K, Hammel G, Bhattacharyya M, de Tomassi A, Nussbaumer T, Neumann AU, Reiger M, Traidl-Hoffmann C: Skin pH-dependent Staphylococcus aureus abundance as predictor for increasing atopic dermatitis severity. Allergy 2020, 75:2888-2898.

18. Korting HC, Lukacs A, Vogt N, Urban J, Ehret W, Ruckdeschel G: Influence of the pH-value on the growth of Staphylococcus epidermidis, Staphylococcus aureus and Propionibacterium acnes in continuous culture. Zentralbl Hyg Umweltmed 1992, 193:78-90.

19. Schommer NN, Gallo RL: Structure and function of the human skin microbiome. Trends Microbiol 2013, 21:660-668.

20. Mukherjee S, Mitra R, Maitra A, Gupta S, Kumaran S, Chakrabortty A, Majumder PP: Sebum and Hydration Levels in Specific Regions of Human Face Significantly Predict the Nature and Diversity of Facial Skin Microbiome. Sci Rep 2016, 6:36062.

21. Glatz M, Jo JH, Kennedy EA, Polley EC, Segre JA, Simpson EL, Kong HH: Emollient use alters skin barrier and microbes in infants at risk for developing atopic dermatitis. PLoS One 2018, 13:e0192443.

22. Mao-Qiang M, Fowler AJ, Schmuth M, Lau P, Chang S, Brown BE, Moser AH, Michalik L, Desvergne B, Wahli W, et al: Peroxisome-proliferator-activated receptor (PPAR)-gamma activation stimulates keratinocyte differentiation. J Invest Dermatol 2004, 123:305-312.

23. Garg A, Chren MM, Sands LP, Matsui MS, Marenus KD, Feingold KR, Elias PM: Psychological stress perturbs epidermal permeability barrier homeostasis: implications for the pathogenesis of stressassociated skin disorders. Arch Dermatol 2001, 137:53-59.

24. Schmuth M, Feingold KR, Elias PM: Stress test of the skin: The cutaneous permeability barrier treadmill. Exp Dermatol 2020, 29:112-113.

25. Zeeuwen PL, Boekhorst J, van den Bogaard EH, de Koning HD, van de Kerkhof PM, Saulnier DM, van S, II, van Hijum SA, Kleerebezem M, Schalkwijk J, Timmerman HM: Microbiome dynamics of human 
epidermis following skin barrier disruption. Genome Biol 2012, 13:R101.

26. Nakatsuji T, Chiang HI, Jiang SB, Nagarajan H, Zengler K, Gallo RL: The microbiome extends to subepidermal compartments of normal skin. Nat Commun 2013, 4:1431.

27. Breternitz M, Flach M, Prassler J, Elsner P, Fluhr JW: Acute barrier disruption by adhesive tapes is influenced by pressure, time and anatomical location: integrity and cohesion assessed by sequential tape stripping. A randomized, controlled study. Br J Dermato/ 2007, 156:231-240.

28. Lademann J, Jacobi U, Surber C, Weigmann HJ, Fluhr JW: The tape stripping procedure-evaluation of some critical parameters. Eur J Pharm Biopharm 2009, 72:317-323.

29. Parra JL, Paye M, Group E: EEMCO guidance for the in vivo assessment of skin surface pH. Skin Pharmacol Appl Skin Physiol 2003, 16:188-202.

30. Berardesca E, Loden M, Serup J, Masson P, Rodrigues LM: The revised EEMCO guidance for the in vivo measurement of water in the skin. Skin Res Technol 2018, 24:351-358.

31. Edgar RC: UPARSE: highly accurate OTU sequences from microbial amplicon reads. Nat Methods 2013, 10:996-998.

32. Camacho C, Coulouris G, Avagyan V, Ma N, Papadopoulos J, Bealer K, Madden TL: BLAST+: architecture and applications. BMC Bioinformatics 2009, 10:421.

33. Wickham H: ggplot2. Elegant Graphics for Data Analysis. New York: Springer-Verlag; 2009.

34. Patil I: Visualizations with statistical details: The 'ggstatsplot' approach. Journal of Open Source Software 2021, 6:3167.

35. Jiang YJ, Lu B, Tarling EJ, Kim P, Man MQ, Crumrine D, Edwards PA, Elias PM, Feingold KR: Regulation of ABCG1 expression in human keratinocytes and murine epidermis. J Lipid Res 2010, 51:3185-3195.

36. Kao JS, Fluhr JW, Man MQ, Fowler AJ, Hachem JP, Crumrine D, Ahn SK, Brown BE, Elias PM, Feingold KR: Short-term glucocorticoid treatment compromises both permeability barrier homeostasis and stratum corneum integrity: inhibition of epidermal lipid synthesis accounts for functional abnormalities. J Invest Dermatol 2003, 120:456-464.

37. Ogai K, Shibata K, Takahashi N, Ogura K, Okamoto S, Sugama J: Amplicon-based skin microbiome profiles collected by tape stripping with different adhesive film dressings: a comparative study. $B M C$ Microbio/ 2021, 21:54.

38. Bagci IS, Ruini C, Niesert AC, Horvath ON, Berking C, Ruzicka T, von Braunmuhl T: Effects of ShortTerm Moisturizer Application in Different Ethnic Skin Types: Noninvasive Assessment with Optical Coherence Tomography and Reflectance Confocal Microscopy. Skin Pharmacol Physiol 2018, 31:125-133.

39. Bouslimani A, da Silva R, Kosciolek T, Janssen S, Callewaert C, Amir A, Dorrestein K, Melnik AV, Zaramela LS, Kim JN, et al: The impact of skin care products on skin chemistry and microbiome dynamics. BMC Bio/ 2019, 17:47. 
Figures

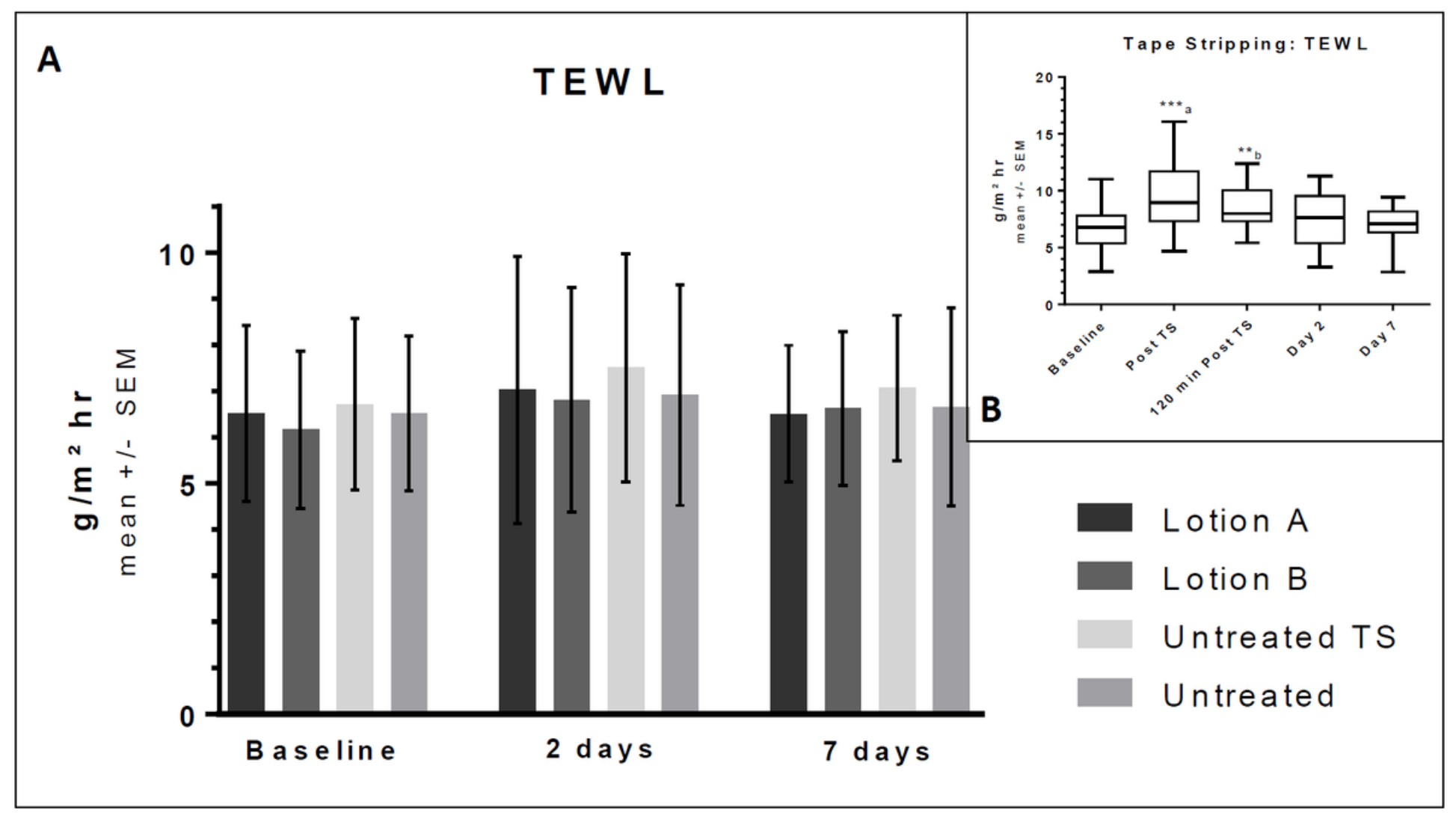

Figure 1

Panel A: Barrier Function (TEWL) in the different treatment groups: no significant differences within time groups and within treatment groups (ANOVA>0.05) Panel B: Tape stripping model (TEWL): ANOVA between time groups $p<0.001$; Sidak's multiple comparisons test: ${ }^{\star \star *}$ a: Post TS vs. Baseline: $p<0.001 ;{ }^{*}$ b: 120 min Post TS vs. Baseline: $p<0.01$; Day 2 and Day 7 vs. Baseline: n.s. 


\section{SC Hydration}

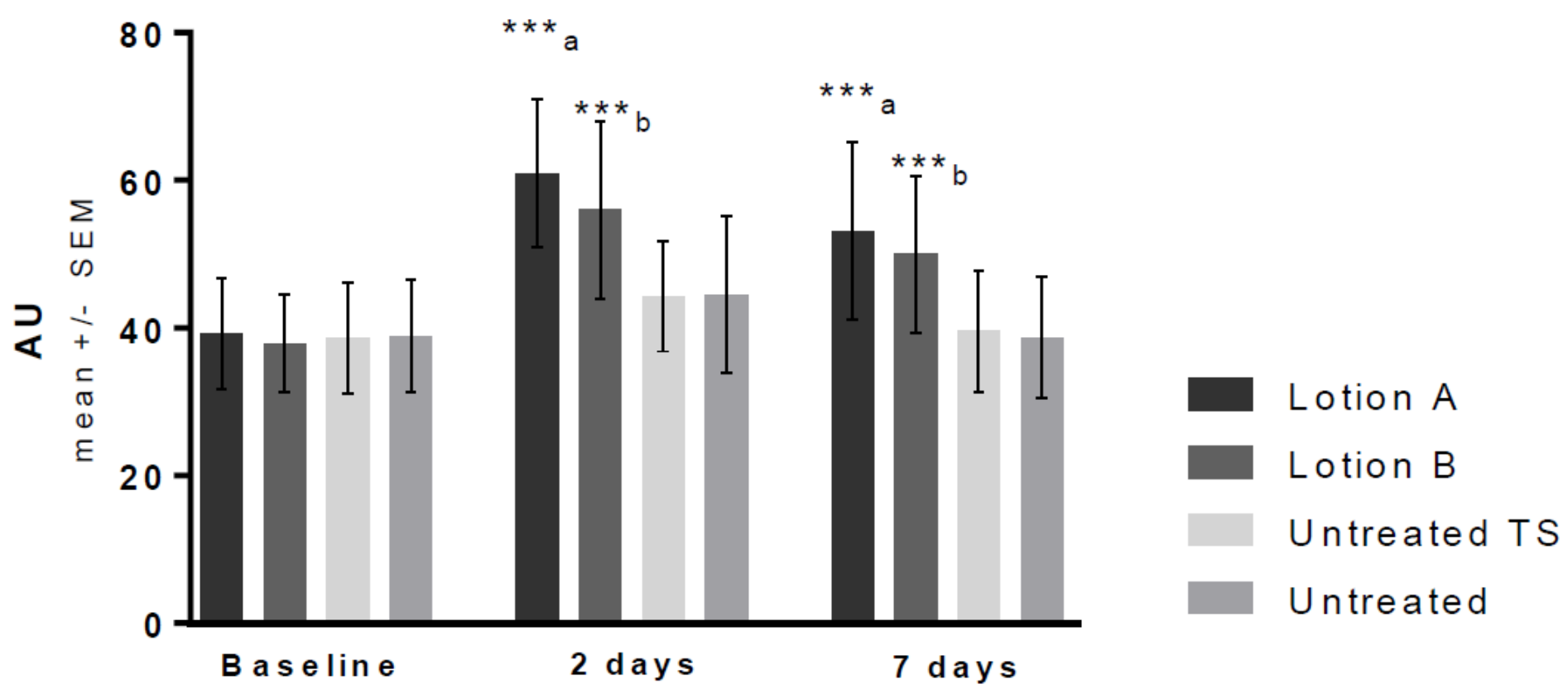

Figure 2

Stratum corneum (SC) hydration: ANOVA within time groups: Baseline=n.s.; both 2 days and 7 days: $p<0.001$; Dunn's multiple comparisons test: ${ }^{\star \star \star} a$ : Lotion A vs. untreated TS: $p<0.001$; ${ }^{\star \star} b$ : Lotion $B$ vs. untreated TS: $p<0.001$; Lotion A vs. B: n.s. [ANOVA within treatment groups: for both lotions $p<0.001$; Dunn's multiple comparisons test; day 2 and day 7 vs. BL $p<0.001$; but n.s. day 2 vs. day 7] 


\section{$\mathrm{pH}$}

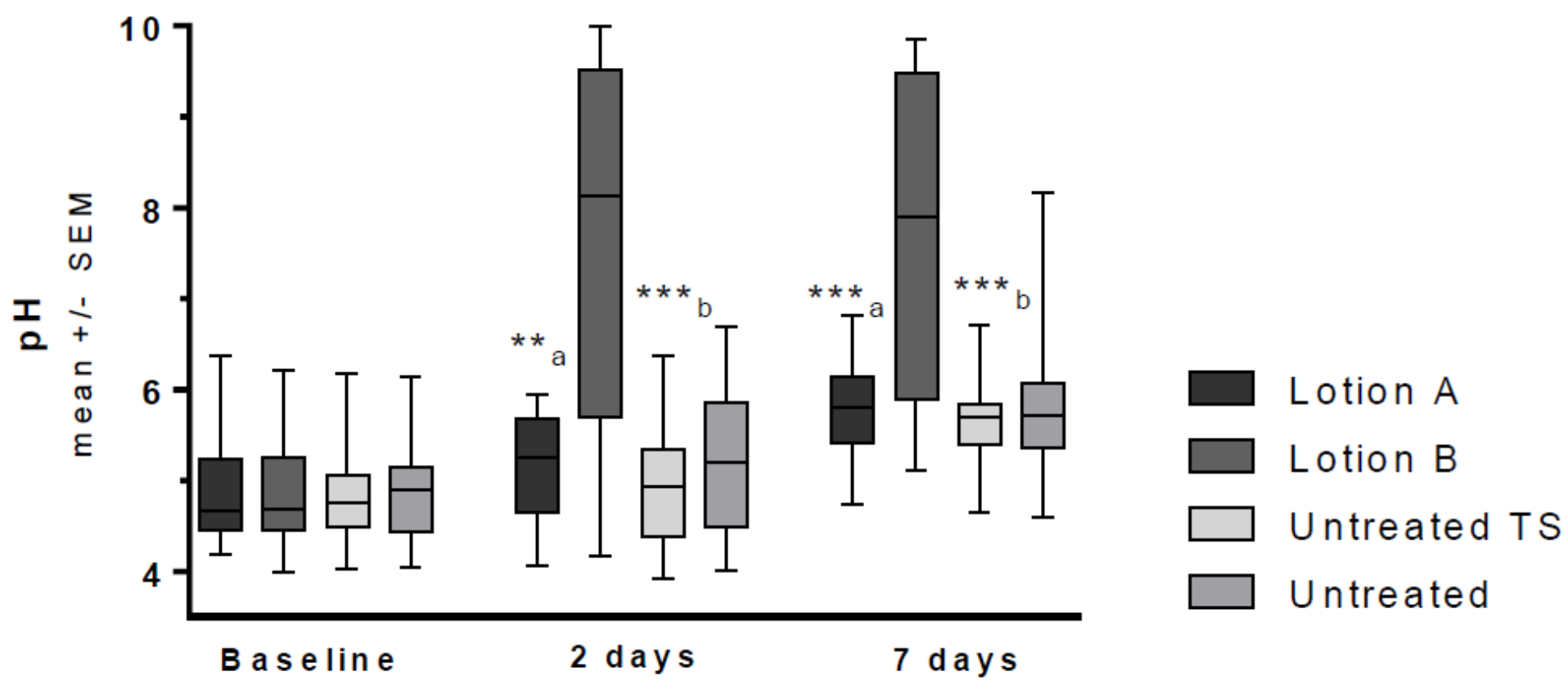

Figure 3

Surface $\mathrm{pH}$ : ANOVA within time groups: Baseline=n.s.; both 2 days and 7 days: $p<0.0001$; Dunn's multiple comparisons test: Day2: **a: Lotion A vs. Lotion B: $p<0.01$; $\star \star \star b$ : Lotion B vs. untreated TS: $p<0.001$;

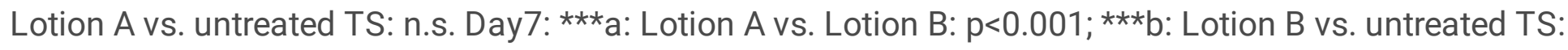
p<0.001; Lotion A vs. untreated TS: n.s. 
A

Control

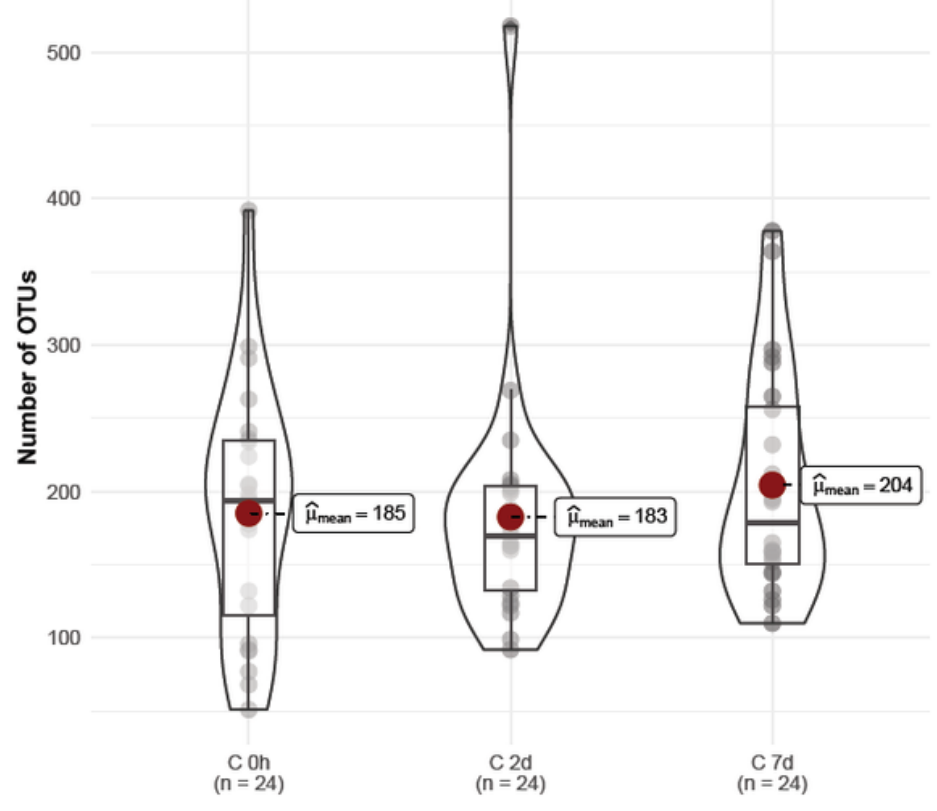

C

2 days after tape stripping

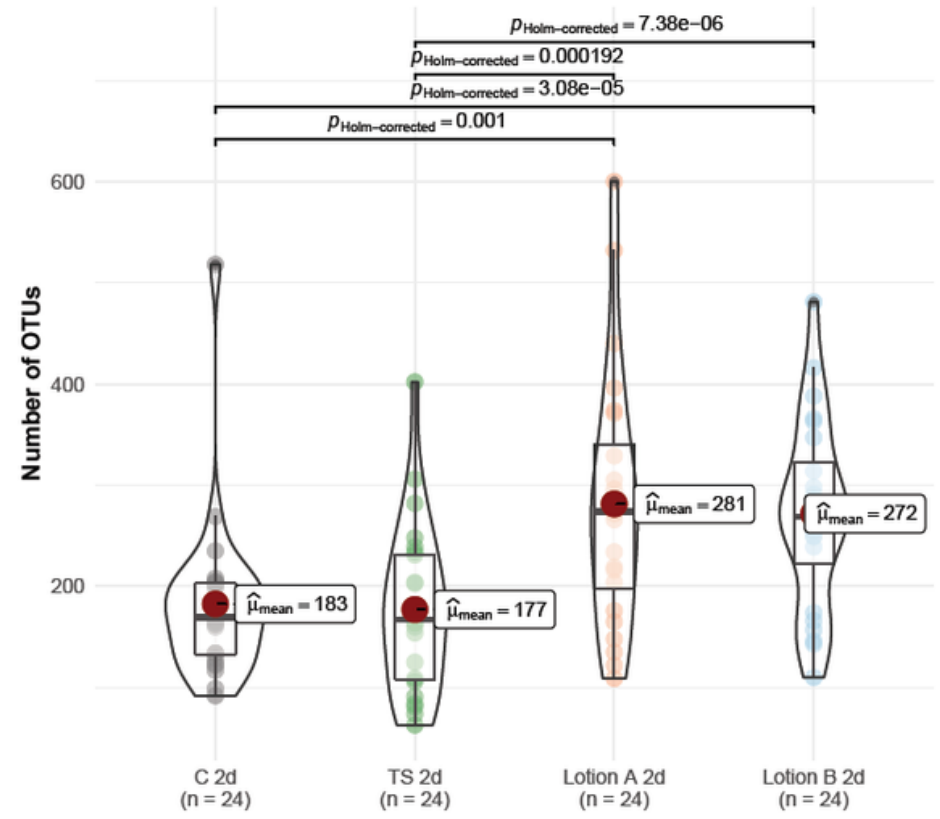

Untreated

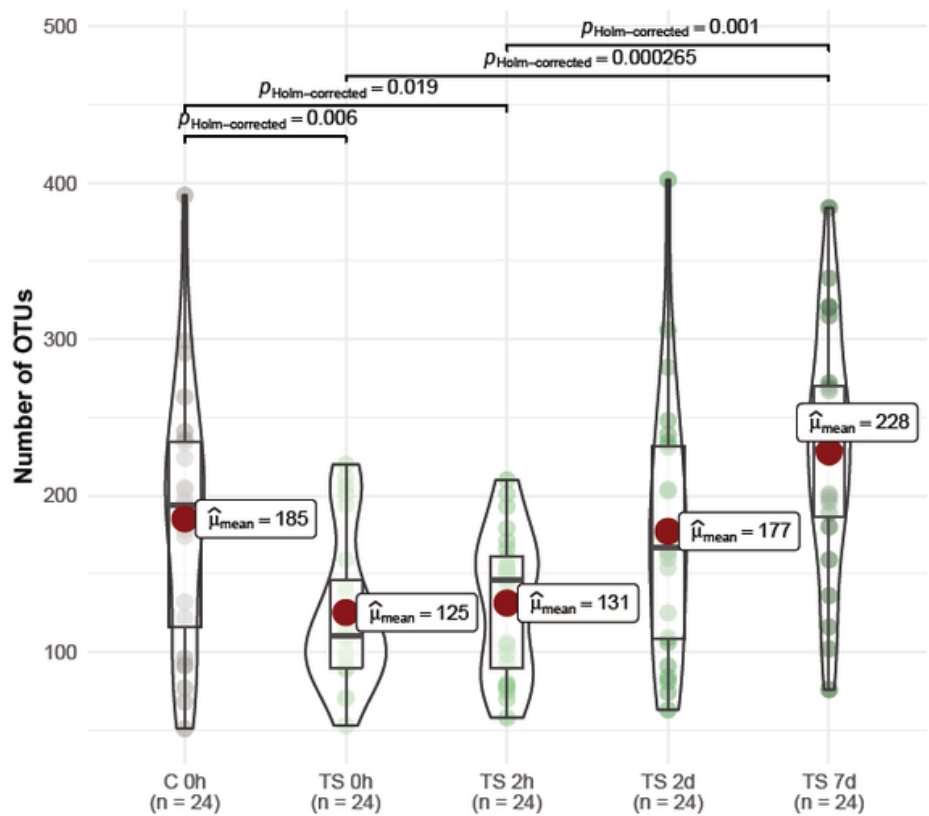

D

7 days after tape stripping

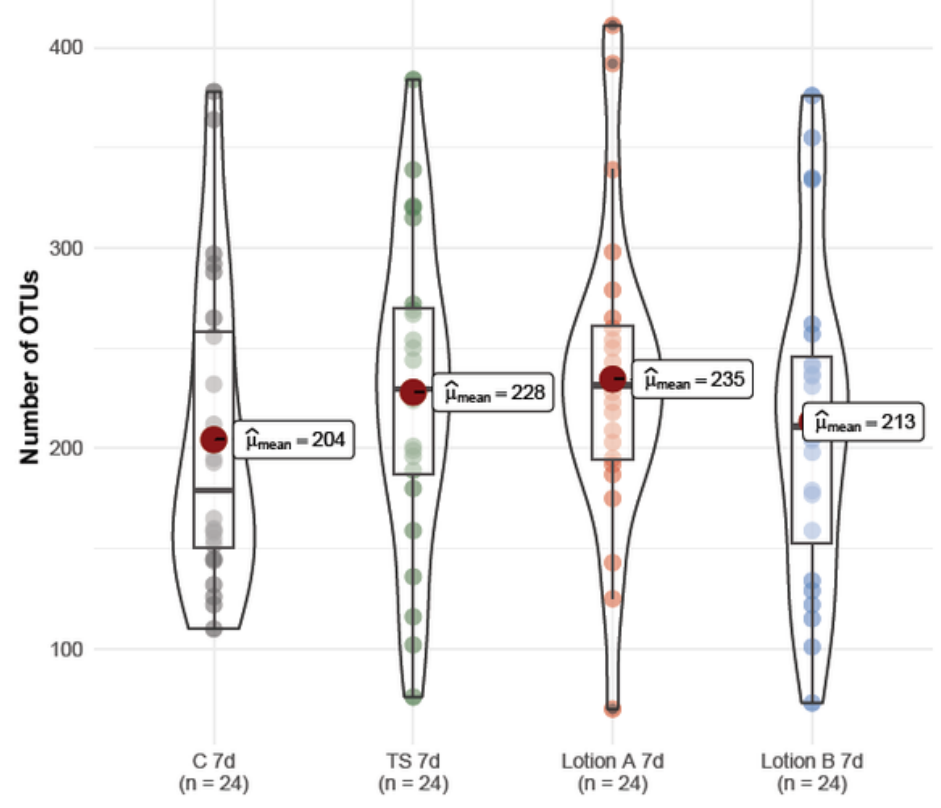

\section{Figure 4}

Distribution of alpha diversity, measured by the number of OTUs between treatment areas. Statistically significant differences are highlighted by P-values. The box plot shows median, $25 \%$ and $75 \%$ percentile, the red dot indicates the mean of each group. Panel a shows the control area over the 7 days without any treatment. No difference was detected in the untreated area. Panel b shows the tape stripped area without treatment. The reduced alpha diversity increased after 2 days (n.s.) and was significantly increased after 7 days ( $p<0.001$ compared to TS 0hrs; $p<0.001$ compared to TS $2 \mathrm{hrs}$ ) Panel c shows all 4 treatment areas 
after 2 days. Both lotions (red dots -3rd violin lotion A; blue dots -4 th lotion B) induced a significant increase of alpha diversity compared to the control (grey dots) and TS area (green dots), $(p<0.001$ and lower) for all comparison. Lotion A shows a slightly higher alpha diversity than Lotion B without reaching the statistical significance. Panel d shows all 4 different treatment areas after 7 days. TS, lotion A and lotion $\mathrm{B}$ are slightly higher than the untreated control area without reaching statistical significance.

A

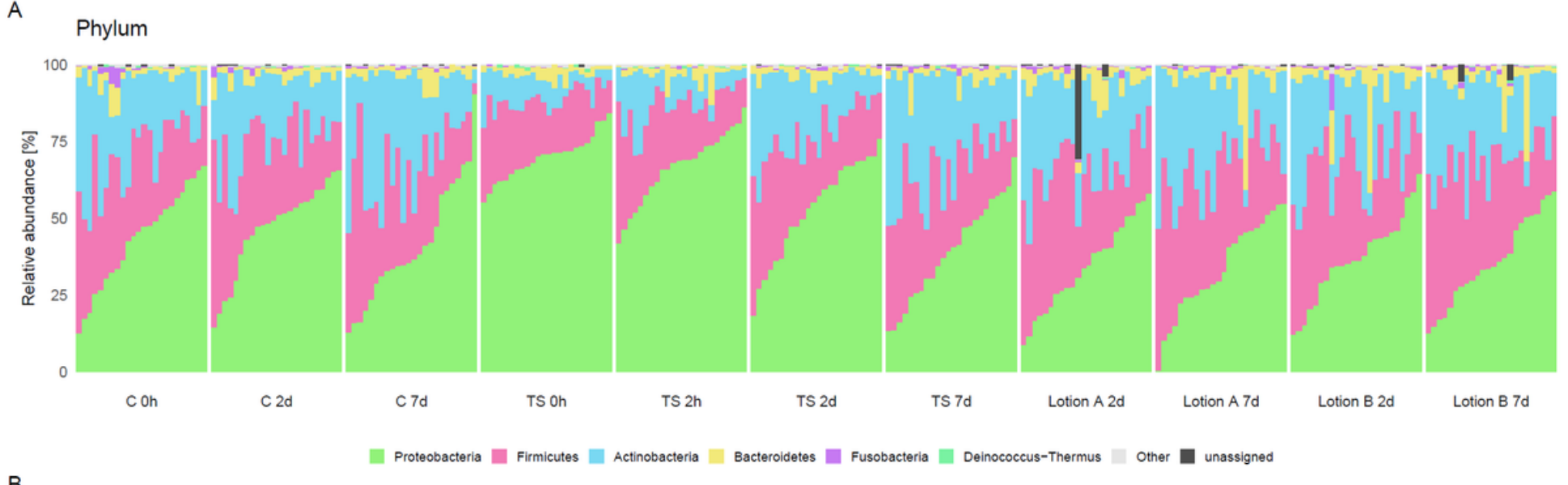

B

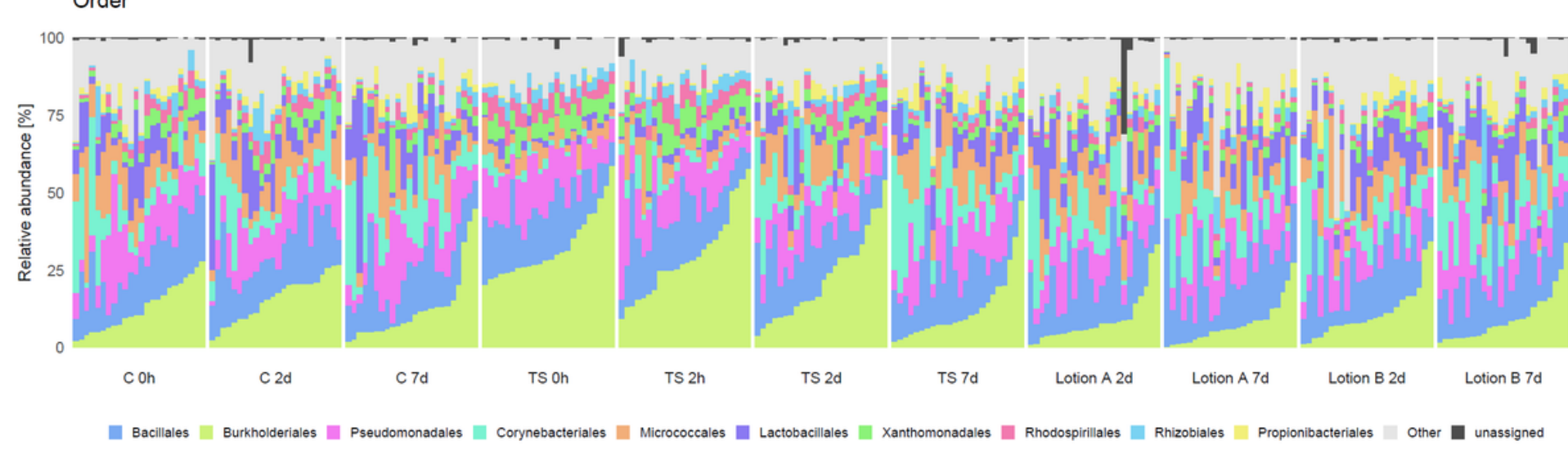

Figure 5

Distribution of the most abundant phyla (a) and orders (b) for each individual, grouped into the different treatment areas at different time points. 


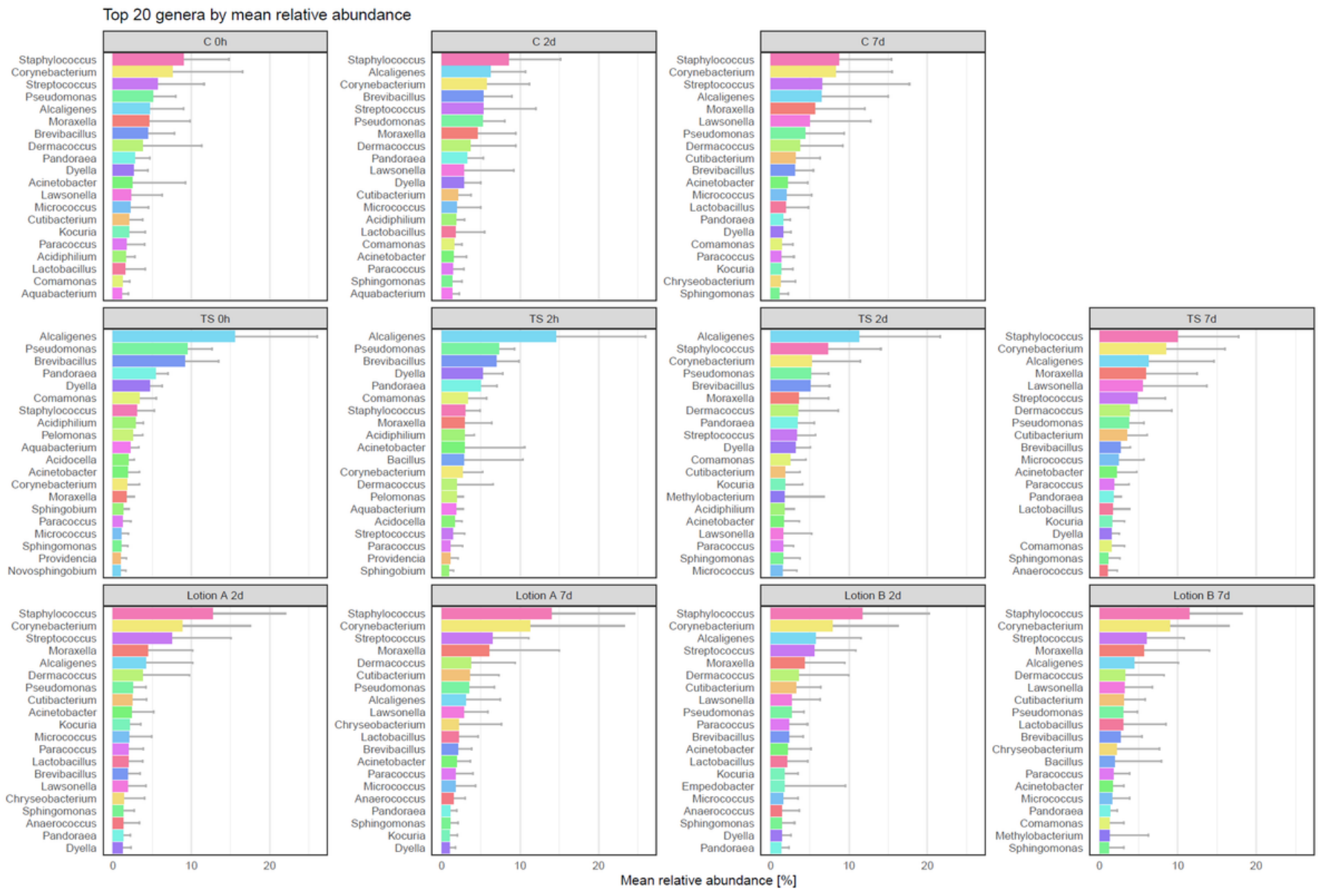

Figure 6

Mean relative abundances of the 20 most abundant genera within the different treatment areas. The first row sows the control areas at baseline, after 2 days and after 7 days. The second row depicts the TS areas immediately after TS, 2 days and 7 days after TS without treatment (the $2 \mathrm{hr}$ time point is shown in row 3). The third and fourth rows show Lotion A and B after 2 days and 7 days, respectively. 
A
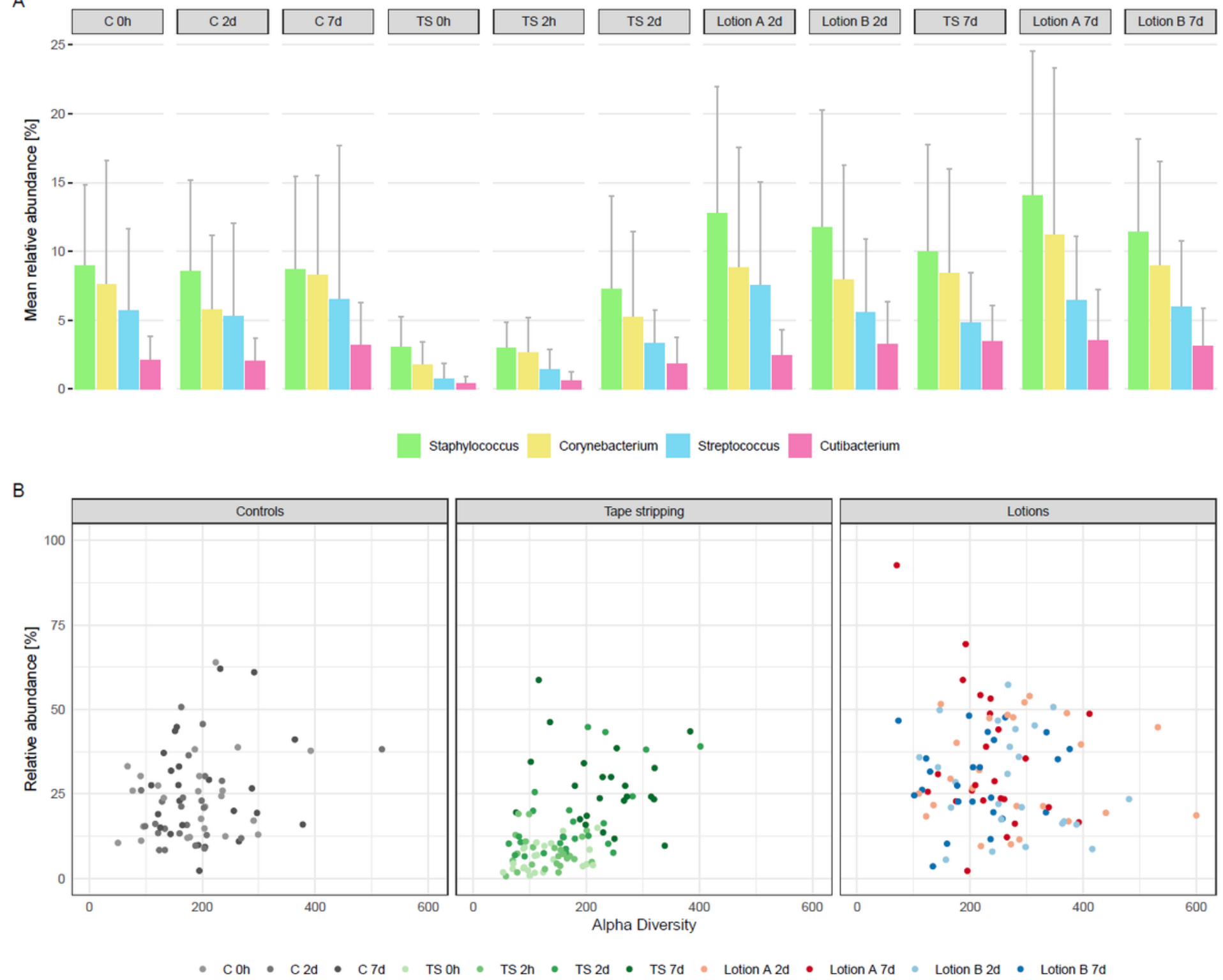

\section{Figure 7}

Panel A: The mean relative abundance of 4 selected genera Corynebacterium, Cutibacterium, Staphylococcus and Streptococcus within the different treatment areas. Panel B: Combined relative abundance of these 4 genera in relation to alpha diversity split into control, untreated and treated areas. Controls (grey dots): No major change in the relative abundance / alpha diversity ratio was detected over time in the control area. Tape stripped areas (green dots): A slight shift of the relative abundance /alphadiversity was induced by TS with lower ratio directly after TS and an increase over time. Treated areas: (Lotion A red dots; Lotion B blue dots). Lotion A and B showed no major difference. Lotion treated (colored dots): at different time points: No major change in the relative abundance / alpha diversity ratio was detected over time in the control area. 


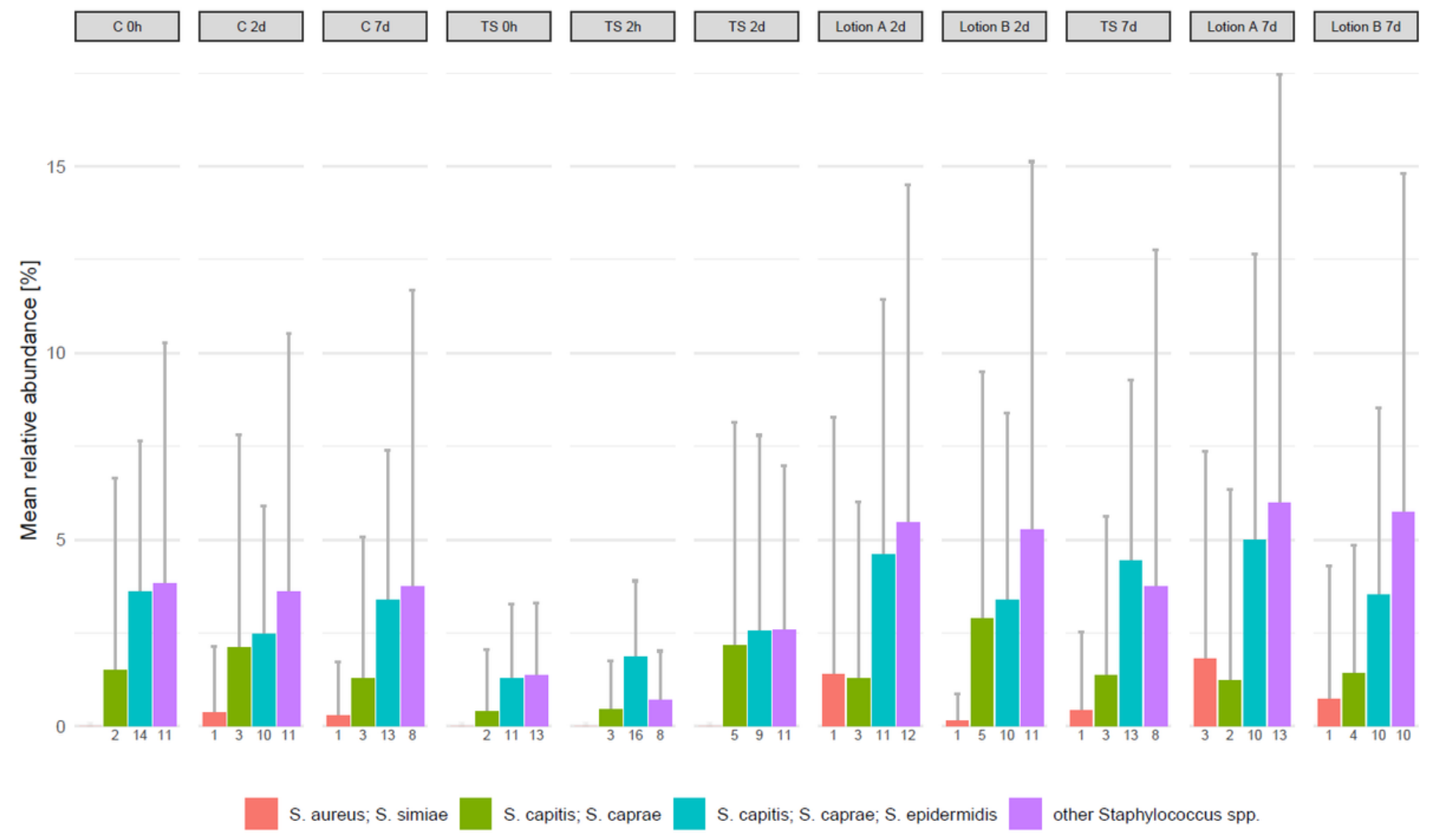

\section{Figure 8}

Relative abundance of OTUs classified to Staphylococcus aureus, S. simae, S. capitis, S. caprae. OTUs are assigned to multiple species, when their V3-V4 sequence is identical. The abundances of other Staphylococcus species are summarized. Numbers below bars denote the number of samples in which this OTU was observed.

Combined relative abundance of Staphylococcus, Streptococcus, Cutibacterium, and Corynebacterium

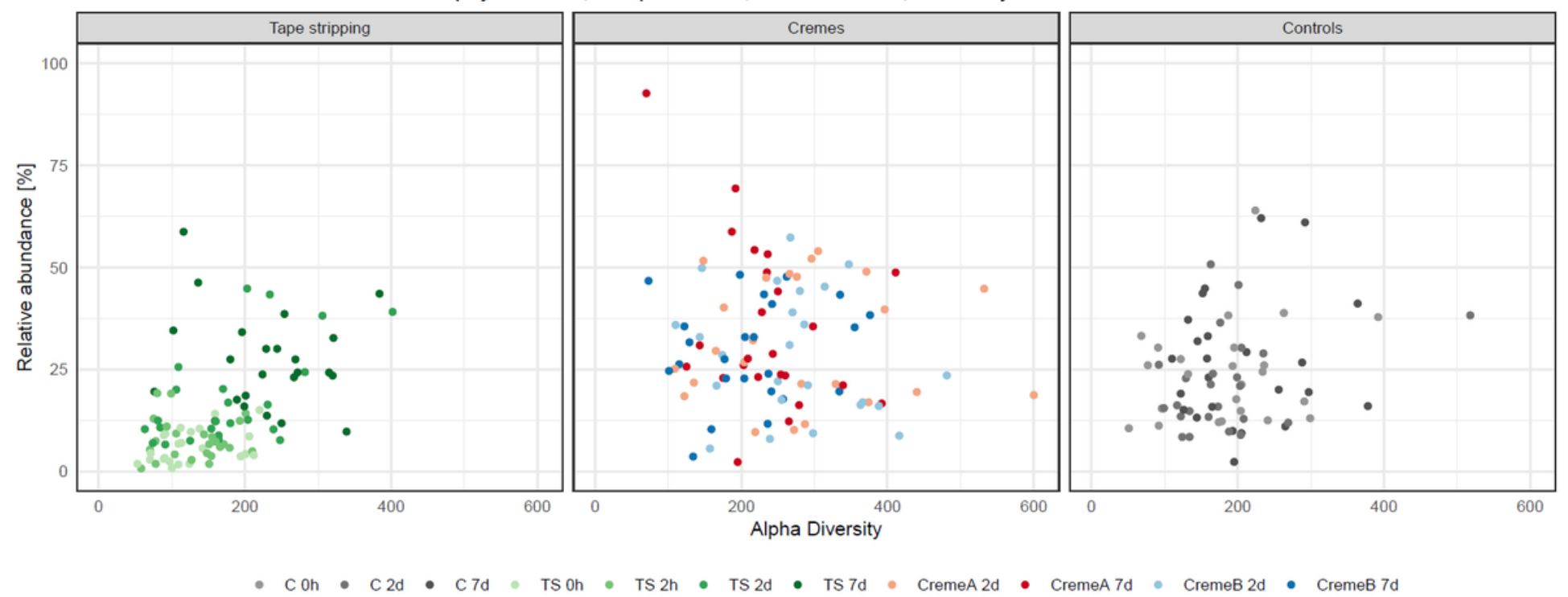

Figure 9 
Legend not included with this version.

\section{Supplementary Files}

This is a list of supplementary files associated with this preprint. Click to download.

- Supplfigures.pdf 